\title{
ionbot: a novel, innovative and sensitive machine learning approach to LC-MS/MS peptide identification
}

\author{
Sven Degroeve t.t, Ralf Gabrielst.f, Kevin Velghet.t, Robbin Bouwmeestert., \\ Natalia Tichshenkot.f, and Lennart Martenst.: \\ †VIB-UGent Center for Medical Biotechnology, VIB, Ghent, Belgium \\ ‡Department of Biomolecular Medicine, Ghent University, Ghent, Belgium \\ E-mail: sven.degroeve@ugent.be
}

ORCID iDs

Sven Degroeve: https://orcid.org/0000-0001-8349-3370

Ralf Gabriels: https://orcid.org/0000-0002-1679-1711

Kevin Velghe: https://orcid.org/0000-0002-9968-6043

Robbin Bouwmeester: https://orcid.org/0000-0001-6807-7029

Natalia Tichshenko: https://orcid.org/0000-0001-7914-0759

Lennart Martens: https://orcid.org/0000-0003-4277-658X 


\section{Abstract}

Mass spectrometry-based proteomics generates vast amounts of signal data that require computational interpretation to obtain peptide identifications. Dozens of algorithms for this task exist, but all exploit only part of the acquired data to judge a peptide-to-spectrum match (PSM), ignoring important information such as the observed retention time and fragment ion peak intensity pattern. Moreover, only few identification algorithms allow open modification searches that can substantially increase peptide identifications.

We here therefore introduce ionbot, a novel open modification search engine that is the first to fully merge machine learning with peptide identification. This core innovation brings the ability to include a much larger range of experimental data into PSM scoring, and even to adapt this scoring to the specifics of the data itself. As a result, ionbot substantially increases PSM confidence for open searches, and even enables a further increase in peptide identification rate of up to $30 \%$ by also considering highly plausible, lower-ranked, co-eluting matches for a fragmentation spectrum. Moreover, the exclusive use of machine learning for scoring also means that any future improvements to predictive models for peptide behavior will also result in more sensitive and accurate peptide identification. 


\section{Main}

Liquid Chromatography combined with high-resolution (Tandem) Mass Spectrometry (LC-MS/MS) has established itself as an invaluable technology for sensitive proteome analysis. It generates vast amounts of raw signal data that require biological interpretation from dedicated bioinformatics tools, known as peptide identification engines. These engines seek to accurately match the observed LC-MS/MS signals with the peptide molecules that generated them. To date, dozens of such identification engines have been developed ${ }^{1}$.

Nevertheless, peptide identification from LC-MS/MS data remains far from trivial. Spectra are noisy and incomplete, and sequences can be several tens of amino acids long. This leads to an extremely large number of potential target sequences, which in turn poses computational challenges, along with specificity challenges ${ }^{2}$. As a result, a commonly employed tactic for identification engines is to reduce the search space to only the proteome of interest for the sample under study. Yet, even this strong restriction is typically not sufficient, as several hundreds of potential amino acid modifications should be considered as well, again leading to an enormous growth in the number of possible targets, even for small proteomes. Most of the classical identification engines maintain a more manageable search space by drastically reducing the amount of potential modifications that can be considered. The obvious result is that many relevant modifications may be missed, leading to many false negatives. Recently, several so-called open modification search engines have therefore been developed, that see to address this limitation by allowing the full range of potential modifications to 
be considered during a fast open modification search that can be applied to large LCMS/MS datasets ${ }^{3-5}$.

All identification engines compute a score for each considered peptide-to-spectrummatch (PSM) that is typically designed to reflect the probability of obtaining a true positive identification. As this PSM scoring function decides on the best match for a given MS2 spectrum, the resulting scores should predict the likelihood of a true positive identification as accurately as possible, especially when searching spectra against large search spaces, as is the case for open searches ${ }^{2}$. With different engines implementing different PSM scoring functions, the set of identified PSMs can be very different between them.

Ideally, the PSM scoring function accurately models the expected LC-MS/MS signal from a given peptide and relies on the comparison between the expected and the observed data to judge a match. This can be achieved in practice by exploiting all accessible PSM data as matching information, including the observed retention time for the LC separation, the precursor $\mathrm{m} / \mathrm{z}$ for the MS1 analysis, and the MS/MS spectrum for the fragmentation analysis. Importantly, Machine learning models already exist to accurately predict calibrated expected retention times ${ }^{6-8}$ and expected MS2 peak intensities from peptide sequences ${ }^{8-11}$

However, current identification engines fail to efficiently exploit this matching information in the scoring function. Typically, the PSM score is computed by counting matched peaks, in some way weighted by the MS2 intensities they explain. This implicitly or explicitly penalizes unmatched fragment ions, which is particularly problematic in open 
searches, where more accurate scoring functions are required. To address this issue and improve accuracy, the relationship between the peptide amino acid sequence and the corresponding peak intensity pattern needs to be considered in the PSM scoring function ${ }^{12}$.

An ideal PSM scoring function would optimally combine all the relevant sources of matching information into a single accurate score. Substantial progress towards building such a scoring function was made by applying Machine learning to rescore leveraged feature vector representations of PSMs that can contain any source of information ${ }^{13,14}$. The PSM rescoring function is then computed from these feature vector representations of the experimental data. However, only limited data is explored as only first-ranked PSMs obtained by an engine are rescored. This means that false first-ranked PSMs cannot be replaced by the true PSM based on the leveraged matching information.

We here therefore introduce ionbot, a completely new type of open modification search engine that exploits the ability to incorporate all relevant matching information into a single, Machine learning-based score. To achieve this, we introduce the concept of a candidate match set that is not restricted to first-ranked matches and is computed from a vast open search space using predicted sequence tags ${ }^{15}$ and a set of biased PSM scoring functions. Furthermore, the ionbot PSM scoring function is computed from this candidate match set using semi-supervised Machine learning, making the PSM scores reproducibly tailored to the experimental data. This leads to an engine that outperforms traditional search engines, allows for reliable open modification searches that outperform current open modification engines, and can be readily adapted to very 
specific conditions, such as TMT labelled data sets, with an even more dramatic increase in identification rates. Finally, we show that our approach naturally leads to the identification of a substantial amount of highly plausible lower-ranked co-eluting PSMs from chimeric MS2 spectra. Throughout, ionbot maintains a tightly controlled FDR, illustrating superior sensitivity while maintaining specificity. 


\section{Results}

\section{Sequence tag prediction models learn from MS2 peak intensities and show high accuracy}

In this section the prefix and suffix tag prediction models (Methods) implemented in ionbot are evaluated on the testing set by the Area under the ROC Curve (AUC) and the Average Precision (AP) computed from the Precision-Recall (PR) curve. All models show very high predictive accuracy with suffix tag models $(A \cup C=99.9 / A P=98.2$ for $\mathrm{HCD}$ and $\mathrm{AUC}=99.9 / \mathrm{AP}=99.4$ for $\mathrm{HCDTMT}$ ) performing better than prefix tag models (AUC=99.8/AP=93.1 for HCD and ACU=99.9/AP=99.2 for HCDTMT) (Supplementary

Fig. 1). It is worth noting that TMT trained models show highest predictive accuracy, especially for the prefix tags.

Furthermore, scoring a HCD testing set with a HCDTMT model and vice versa substantially decreases predictive accuracy. For the models trained on HCD and evaluated on HCDTMT, the prefix model reduced to $A U C=98.9$ and $A P=58.7$, while the suffix model shows a slight decrease to $A U C=99.8$ and $A P=95.6$. Notably, for models trained on HCDTMT and evaluated on HCD, the prefix models prediction performance decreased much further to $A \cup C=87.7$ and $A P=7$, with a smaller decrease for the suffix model $(A \cup C=99.5 / A P=92.2)$.

To further evaluate the models, the true PSMs identified by an open search were analyzed. For each true PSM a prefix and suffix tag ranking was computed by scoring 
all tags with the corresponding predictive models. The highest rank between the true prefix and suffix tag (determined by the matched peptide) is then recorded as a metric for how well the predicted sequence tags can reduce the search space (Methods). The vast majority of these ranks were within the top-10, with many ranked first for one of the two models (Supplementary Fig. 2).

\section{Expanding the search space is crucial; we recommend to no longer use closed}

\section{searches}

Open searches can match peptidoforms never considered in closed searches.

However, at the same time, a larger search space leads to higher scoring decoy

matches, thereby potentially increasing the PSM score threshold required to maintain a $1 \%$ FDR $^{16}$. In this section we investigate the difference between a closed and open ionbot search for the five evaluation datasets (Methods).

Our findings confirm previous research showing that open searches considerably increase proteome coverage. The increase in PSM identifications is up to $56 \%$ for HEK239 (Fig. 1a), and even 74\% for TMTCPTAC (Supplementary Fig. 3). At the unique peptide level, identification gains go up to $20 \%$ (HEK239). It is worth noting that this overall increase in the number of identifications will play an important role in the accurate downstream protein inference and quantification, as well as in increasing the power of the succeeding differential analyses.

Counting PSM and peptide identifications does not reveal all the differences between a closed and open search. We found that a substantial number of closed search 
identifications are no longer called in the corresponding open search. This is $11 \%$ of the closed search identifications for HEK239 (Fig. 1b) and goes up to $21 \%$ for the Breast dataset (Supplementary Fig. 5). Furthermore, many of these 'lost' matches are overruled by a better match in the open search, as indicated in the figures. It is likely that most of these overruled peptide matches are incorrect and have been forced upon closed search identifications due to the absence of the otherwise higher scoring, true peptide ${ }^{17}$.

Notably, the majority of PSMs gained in open searches are explained by the wide (7.5Da) precursor mass error tolerance that ionbot allows for matches without unexpected modification. These precursor errors show a periodic pattern at $1 \mathrm{Da}$ intervals (Supplementary Fig. 6). It is therefore possible that incorrect peak picking at the isotope level accounts for these.

\section{Prediction models trained on specific experimental conditions improve identification}

For each predictive model implemented in ionbot (tag-models, MS2PIP and DeepLC), there is a version trained on unlabeled HCD data, and a version trained on TMT labeled HCD data. In this section we apply ionbot with TMT specific prediction models on the non-TMT labeled evaluation datasets. Similarly, we applied ionbot not using TMTspecific models on the TMTCPTAC dataset.

We observed a 19\% decrease in PSM and a 16\% decrease in peptide identifications when employing non-optimal predictive models in HEK239 (Fig. 1a). For the other 
datasets the loss amounts $16 \%$ for CD8T, $18 \%$ for Brain, $26 \%$ for Breast, and $16 \%$ for TMTCPTAC. At the peptide level these losses are repeated, with a loss of $13 \%$ for CD8T, 17\% for Brain, 22\% for Breast, and 20\% for TMTCPTAC (Supplementary Fig. 34).

\section{Predicted retention time and fragment ion intensities provide decisive PSM information}

In this section we investigate the relevance of the DeepLC retention time predictions (RT-pred-error) and MS2PIP peak intensity predictions (intensity-correlation) features in the ionbot PSM scoring function (Methods). Grouping PSMs by peptidoform (Methods) to compute the corrected observed retention time to compute RT-pred-error clearly reduced long elution time windows peptidoforms identified by multiple spectra (Fig. 1de). This is especially true for peptides at the end of an LC run, where the issue can be even more problematic for the RT-pred-error feature.

Comparing open searches with and without using the RT-pred-error feature in the PSM scoring function showed that consistently more PSMs were identified when the feature is added to the scoring function. At first, this gain appears to be relatively small, at $3.2 \%$ for HEK239 (Fig. 1a) and 2.4\% for CD8T, 2.9\% for Brain, 7.2\% for Breast, and 1\% for TMTCPTAC (Supplementary Fig. 1), but it is worth noting that the vast majority of true PSMs are (also) confirmed by the other sources of matching information, which leaves the retention time feature to correct only ambiguous situations that cannot be distinguished by any of the other sources. We found that PSM identifications unique to the search not using RT-pred-error show high retention time error in general, and, that 
many PSMs that were overruled when adding the feature show high prediction error as well (Fig. 1f, Supplementary Fig. 8).

Similarly, we compared open searches with and without using the intensity-correlation feature in the scoring function. The latter search also does not use this correlation information as a biased PSM scoring function. We saw an increase in PSMs (6\%) identified when adding the feature for HEK239 (Fig. 1a). For the other datasets the gain is $5 \%$ for CD8T, $6 \%$ for Brain and Breast, and $8 \mathrm{~s} \%$ for TMTCPTAC (Supplementary Fig. 1). Yet here again, this information only gains importance in ambiguous situations that cannot be distinguished by any of the other sources. For HEK239, identifications called only by using the intensity-correlation feature show high correlations (Fig. 1g), while PSMs that are eliminated when the correlation feature is used show low overall correlations (Fig. 1i). Also, for overruled matches when intensity-correlation is used, the difference in correlation can be large, even though the vast majority shows only small differences in the higher correlation range (Fig. 1h). In these cases, it becomes difficult to decide on the correct match based on correlation and other matching information is required to decide on the true match. The same conclusions were made for the other evaluation datasets (Supplementary Fig. 9).

We repeated the same experiment with $\mathrm{RT}$-pred-error and intensity-correlation omitted from the scoring function. This resulted in a much more substantial increase in the number of PSM identifications, with 11\% for HEK239 (Fig 1a), 9\% for CD8T, 11\% for Brain, 16\% for Breast, and 10\% for TMTCPTAC (Supplementary Fig. 1). At the peptide 
level the gains amount to $9 \%$ for CD8T, $12 \%$ for HEK239, $14 \%$ for Brain, $12 \%$ for

Breast, and 15\% for TMTCPTAC (Supplementary Fig. 2).

Considering retention time error and intensity correlation in the PSM scoring function not only increases the number of identifications, but also corrects and overrules incorrect matches based on the additional matching information that becomes available. For instance, for HEK239, 2.8\% of the PSMs identified omitting both features were overruled by a better match when using them (Fig. 1c). Similar results were found for the other datasets (Supplementary Fig. 10).

\section{Entrapment peptides confirm accuracy and stability of the ionbot open search FDR estimates}

For the FDR estimates to be meaningful, the ionbot PSM scoring function should treat false matches against the decoy and target database equally, i.e., decoy matches should be representative random matches. For ionbot, this conveys that the PSM scoring function learned from experimental data should not be biased towards favoring matches against the target database.

To estimate a potential matching bias, we adopted the entrapment peptides approach (Methods). If a bias exists, we should observe more than the expected number of matches against the entrapment compared to the decoy database. In our experiment, there are about $10 \%$ more decoy peptides compared to entrapment peptides, so we expect to see this difference in the data. For the CD8T and HEK239 datasets, we observed about $6.2 \%$ and $6.6 \%$ more decoy matches respectively (Supplementary Fig. 
11). As this is well below $10 \%$, we conclude that the accuracy of the FDR estimates is high, implying that the ionbot PSM scoring function is not biased towards matches against the target database.

\section{The ionbot engine compares favorably to other state-of-the-art open modification engines}

Many open search engines exist, but few can produce sensitive identification results for large datasets that contain hundreds of thousands of spectra, mainly due to computational limitations. Two recent engines stand out in terms of performance: MSFragger and open-pFind (Methods).

At the PSM level, MSFragger is slightly more sensitive than open-pFind (Fig. 2a, Supplementary Fig. 12), mainly due to the 7.5Da wide error matches (data not shown) that are considered only in ionbot and MSFragger. At the peptide level, these differences become much smaller, with no obvious ranking for the identification engines. However, plotting PSM and peptide identification overlap reveals a notable level of disagreement between the search engines (Fig. 2b, Supplementary Fig. 13).

To obtain more insight into this disagreement, we looked at the intensity-correlation computed for PSMs uniquely identified by one of the engines. To avoid discussion about the unknown effect of specific modifications on the peak intensity pattern, we limited this investigation to identifications without an unexpected modification. We found that many identifications unique to open-pFind and/or MSFragger are questionable. For HEK239 (Fig. 2c), when we look at the PSMs unique to ionbot, the $25 \%$ with the lowest 
intensity-correlation still show correlation within [0.52,0.77] (excluding outliers). For the PSMs unique to open-pFind and MSFragger this interval is $[0.12,0.58]$ and $[0.15,0.57]$ respectively. The same observations were made for the other datasets, most extreme for TMTCPTAC with more than $25 \%$ of the matches in open-pFind and MSFragger showing a correlation less than 0.59 and 0.63 respectively. While for ionbot, all matches (except very few outliers) have correlations higher than 0.68 (Supplementary Fig. 14).

For PSMs identified by just two engines, the intersection between ionbot and openpFind shows highest intensity-correlation values, while the intersection between openpFind and MSFragger clearly shows the lowest correlations, with $25 \%$ of these identifications having a correlation withing $[0.15,0.61]$ for the HEK239 dataset (Fig. 2c).

For the RT-pred-error information these differences are much smaller (Supplementary Fig. 15). For the intersections there are no obvious differences. But for the matches unique to each engine, the median retention time error for ionbot tends to be half of the median retention time error observed in the other engines.

\section{Identification sensitivity is substantially increased by considering lower ranked, highly plausible co-eluting matches}

To determine the best match for an MS2 spectrum, ionbot learns the PSM scoring function from the candidate match set and then selects the first-ranked match for each spectrum based on the computed scores. Next, a more accurate PSM score is computed from these first-ranked matches, and the statistical significance is determined for these first-ranked PSM scores (Methods). 
Nevertheless, the candidate match set explicitly contains multiple candidates for many spectra (Methods). In this section we investigate computing the statistical significance of scores from all PSMs in the candidate match set, which can then result in multiple candidate peptides passing the 1\% FDR threshold for a given spectrum. We found that even though the FDR threshold does not impose a limit on the number of possible matches, the vast majority of spectra with multiple identified matches had just two (Supplementary Fig. 16). The maximum number of different matches observed for an MS2 spectrum was six.

Next, we focused on those MS2 spectra that had exactly two matches passing the $1 \%$ FDR threshold. For each such spectrum, we computed the edit distance (Levenshtein distance) between the two matched peptide sequences. Fig. 3a plots these edit distances for HEK239 and reveals a clear bimodal distribution, with one distribution tightly centered around distance 1 . The exact same observation was made for the other datasets (Supplementary Fig. 17). PSMs in this distribution are examples of highly similar peptide matches that either co-elute, or that cannot be distinguished using the available matching information. Table 1 shows representative examples of PSMs with edit distance 1 (IDs 1-5). For instance, for the spectrum with ID=1 there is a difference of one amino acid between the two peptide matches, with the difference in mass compensated by a methylation. 
We then continued to investigate matches which we consider to be highly plausible coeluting PSMs (edit distance > 2). In Table 1, the spectra with IDs 6 to 11 show very dissimilar co-eluting PSMs, all with low RT-pred-error and high intensity-correlation. Table 2 shows examples of spectra with four or even five different, highly plausible coeluting matches, each providing potential evidence for different proteins. Universal Spectrum Identifiers ${ }^{18}$ to spectrum annotations can be found in the Supplementary Notes. These further confirm the high plausibility of these co-eluting matches.

Considering all co-eluting matches (edit distance > 2) greatly increases identification sensitivity. For instance, for the HEK239 (Fig. 3b) and Brain (Supplementary Fig. 18) datasets, more than $26 \%$ additionals unique peptide sequences were identified.

For HEK239, comparing different sources of matching information between the firstranked PSMs and the lower-ranked co-eluting PSMs showed that the number of b-and y-ions matched is slightly lower for lower-ranked PSMs (Fig. 3c). However, lowerranked PSMs also tend to be smaller in length (Fig. 3d). For intensity-correlation (Fig. 3f) and RT-pred-error (Fig. 3g), the lower-ranked PSMs again show highly plausible values as compared to the first-raked PSMs. The difference is much more pronounced when we look at the intensity explained by the b- and y-ions (Fig. 3e), which is much lower for the lower-ranked PSMs, resulting in a lower PSM score (Fig. 3h). Nevertheless, based on the other sources of matching information, these PSMs are still highly plausible matches. The same conclusions could be made for the other datasets (Supplementary Fig. 19). 
Finally, we found that a substantial percentage of lower-ranked co-eluting matches were identified as first-ranked match by open-pFind and/or by MSFragger. For HEK23 we found that this was $42 \%$ (Fig. 3i). For the other datasets this was $42 \%$ for CD8T, $41 \%$ for Brain, 34\% for Breast, and 30\% for TMTCPTAC (Supplementary Fig. 20a). We also found that for PSMs unique to ionbot, the vast majority of matched peptides were identified as a first-ranked PSM by open-pFind and/or by MSFragger, but from another MS2 spectrum. For HEK239 this was 77\% (Fig. 3j). For the other datasets this was $68 \%$ for CD8T, $77 \%$ for Brain, $62 \%$ for Breast, and $72 \%$ for TMTCPTAC.

We believe these findings provide strong evidence for the presence of co-eluting PSMs, and that it is remarkably straightforward to discover and study these in ionbot due to its data-driven peptide identification approach. 


\section{Discussion and conclusion}

We presented ionbot, a novel type of open identification engine that is unique in taking full advantage of the accurate predictions provided by Machine learning algorithms. This is realized by omitting the predefined PSM scoring function altogether, and instead relying on a data-driven strategy to learn the weights of all matching information provided which includes the MS2 peak intensity pattern correlation and LC retention time prediction error. This provides ionbot with the flexibility to adapt the PSM scoring function to the experimental conditions and passes the identification bias from the expert to the data.

We have shown that ionbot exploits the additional matching information in its PSM scoring function to increase the number of identifications at the PSM as well as at the peptide level, and that it also allows ionbot to adapt to specific experimental protocols such as TMT-labelling with ease. Moreover, we show that the additional matching information is also highly useful in reducing false positive matches by either eliminating these, or by replacing them with higher scoring identifications.

When compared to other open modification search engines, ionbot performs on par at the identification sensitivity level. However, looking at the PSM evidence in terms of matching information reveals that many identifications unique to the other engines show very low intensity pattern correlation or high LC prediction error. This can indeed be expected as other open search engines do not exploit these sources of matching 
information in their scoring function and, as such, are unable to make accurate decisions based on these sources.

Finally, co-eluting lower-ranked PSMs arise naturally from the data-driven identification strategy implemented in ionbot. Even though many of these lower-ranked matches were considered potentially ambiguous highly similar matches to the first-ranked match, a substantial number were shown to be actual highly plausible co-eluting matches. By considering all these co-eluting matches, ionbot can provide substantially higher identification sensitivity compared to the other open search engines.

We believe this research opens up new avenues for improving the analysis of LCMS/MS data. The Machine learning models employed by ionbot can likely still be improved, especially in terms of predicting the effects on analyte behavior of different protein modifications. Moreover, different (non-)linear models can be evaluated for learning the PSM scoring function. Because ionbot can very easily be fitted with new or improved models, and because ionbot is implicitly highly adaptive, any such improvements will very likely result in more accurate PSM scoring functions. Finally, this highly adaptive nature of ionbot can also allow dedicated optimization for specific experimental conditions, thereby increasing identification sensitivity and accuracy even further. 


\section{Methods}

\section{The search space}

Observed MS2 spectra are matched against a peptide search space that is computed in silico from a known proteome using a specific simplified enzymatic cleavage pattern (e.g., for trypsin). In the Human proteome dataset used in this research (see section Datasets), cleaving after lysine or arginine results in 512.990 unique peptide sequences. This number increases to 2.148 .714 by allowing for two missed cleavages.

Furthermore, proteins can undergo chemical modifications, with some modifications observed more frequently (e.g. oxidation) than others ${ }^{19}$. Public repositories that list previously observed modifications, such as unimod.org, currently contain more than 1000 different modifications that are known to alter a specific amino acid in a peptide. Therefore, the peptide search space should be expanded by all possible modified versions of each peptide. Each modification changes the $\mathrm{m} / \mathrm{z}$ pattern of the MS2 spectrum generated by the modified peptide and can, potentially, also alters the MS2 peak intensity pattern ${ }^{20}$ and expected LC retention time ${ }^{6,21}$. To make matters worse, candidate matches for an MS2 spectrum can become very hard to distinguish due to the expanded search space ${ }^{2}$

The vast majority of peptide identification engines consider only a few of the most expected protein modifications. This is known as a closed search. In contrast, in an open search one tries to consider all possible peptidoforms (considering all possible modification patterns for a peptide) by adding these to the search space. Even though 
this space is still considerably smaller than the one evaluated by de-novo identification engines (which consider all possible peptide sequences as well), as the number of modifications taken into account increases, the search space expands exponentially, putting a strong computational load on the peptide identification task.

To reduce this computational burden, ionbot limits the open search by setting a maximum on the number of modifications allowed within one peptide. In a closed search, the space consists of all peptidoforms that carry at most two expected (variable) modifications simultaneously. Next, to an unlimited number of expected modifications that are fixed. In an open search, the space is further expanded by all peptidoforms that can be constructed by (i) adding one of the $1000+$ post-translational, chemical derivative, or artefactual peptide modifications listed in the unimod.org repository, (ii) considering the delta-masses generated by the modifications in (i) only at the MS1 level, (iii) adding a single amino acid substitution, or (iv) by removing any number of $\mathrm{N}$ terminal amino acids from the peptide to obtain a semi-tryptic peptide. For (i) and (iii), all possible unmodified locations for the modification or substitution are considered. For (ii) ionbot tries to match delta-masses observed only at the MS1 level and therefore not part of the MS2 fragmentation spectrum. For (iv), semi-tryptic peptides are considered up to a length of seven amino acids.

\section{The candidate match set}

The PSM scoring function in ionbot is learned from a candidate match set that is a small subset of the search space, while still being large enough to still contain the true PSMs and to learn an accurate scoring function. To achieve this, ionbot employs a sequence 
tag strategy driven by Machine learning scoring models, and a set of biased expertdriven PSM scoring functions.

A sequence tag is a short amino acid sequence with a prefix mass and a suffix mass that allocates its position within a peptide. Tags are typically computed from a graph representation of an MS2 spectrum ${ }^{22,23}$. This spectral graph exploits fragment ion mass differences but ignores the relationship between the tag's amino acid sequence and the observed MS2 peak intensity pattern. Intensity information is typically exploited only to prefer tags that match higher-intensity peaks.

Instead, ionbot implements a data-driven approach to construct constrained sequence tags. By constraining the tags to the first three (prefix) or the last four (suffix) amino acids in a tryptic peptide, predictive Machine learning models can accurately score a tag from a feature vector representation of the tag and the corresponding MS2 spectrum, thereby eliminating time consuming spectral graph construction, while also exploiting MS2 peak intensity information. For each observed MS2 spectrum, the prefix model scores 8000 3-mers and the suffix model scores 16.000 4-mers, all constructed from nineteen amino acids - structural isomers leucine and isoleucine are treated as a single residue - and oxidized methionine, which was added due to its high prevalence. For each MS2 spectrum, the $T$-top scoring tags from both the prefix and the suffix model are used to reduce the search space to those peptides that match any of these tags within a user-defined MS1 mass error tolerance. For candidate peptides with only fixed, expected or no modifications, this mass error tolerance is increased to 7.5Da, as justified in the Results section. The parameter $T$ should be chosen large enough such that only unlikely peptides are removed from the search space. Here, we 
set $T=50$ as increasing $T$ resulted in just a small identification increase (1\%-3\%) for all datasets while substantially increasing compute time.

The implementation of a set of biased PSM scoring functions further differentiate ionbot from traditional identification engines. These biased functions each consider just one source of matching information and are listed in Table 3. For peak counting and explained intensity information, ionbot also considers different subsets of fragment ion types. The candidate match set is then further reduced to the top-ranked matches for each biased PSM function, except for the intensity correlation function for which ionbot considers the top-3 ranked matches.

\section{The prefix and suffix tag prediction models}

This section explains how the prefix and suffix models score the corresponding 3-mer and 4-mer tags. Both are two-class classification models trained on publicly available spectral libraries (see section Datasets). For a given MS2 spectrum and tag, each model computes the probability that the $k$-mer matches the peptide that generated the spectrum. For the prefix model this is the 3-mer corresponding to the $\mathrm{N}$-terminal three amino acids in the peptide, and for the suffix model this is the 4-mer corresponding to the C-terminal four amino acids in the peptide. The feature vector representation implemented in these models contains discriminative information about both the spectrum and the tag. This allows for modelling the relationship between the tag's amin acid sequence and the observed MS2 peak intensities.

To represent the tag, a one-hot-encoding of the k-mer amino acids was considered, but this yielded much inferior results. Instead, as implemented in our MS'PIP tool ${ }^{11}$, amino 
acids in the $k$-mer are encoded by each of five amino acid properties: mass, hydrophobicity, helicity, basicity and iso-electric point estimates.

The MS2 spectrum is represented by the observed peak intensities, normalized by total ion current of the spectrum ${ }^{24}$, of the relevant fragment ions that can be explained by the tag. These are the first three a-, b-, and c-ions for the prefix model, and the first four $\mathrm{x}_{-}$, $y$ - and z-ions for the suffix model, both with variable $\mathrm{H}_{2} \mathrm{O}$ - and $\mathrm{NH}_{3}$-losses, and with fragment ion charge states $1+$ and 2+ considered as well. Variable methionine oxidation is also encoded by adding three binary features that indicate the presence of an oxidation at any position in the tag (excluding the last amino acid in the suffix model tag, which is restricted to lysine or arginine). Lastly, the precursor charge state and precursor mass are encoded as features as well.

Each PSM in the training set is represented by a feature vector for each model, which are collectively labeled as the positive class. For the negative class, the MS2 spectrum is matched to $N$ different random $k$-mers from which features vectors are created. For the suffix model, these negative examples remain constrained to lysine or arginine at position four. We found $N=200$ to be a sufficient number of negative $k$-mer examples, while balancing with the computational cost of computing the models.

As in MSPIP, accurate predictive gradient boosted tree models (GBT) are learned with the open-source XGBoost tool ${ }^{25}$. The boosting algorithm fits an additive decision tree ensemble on the data that allows for extremely fast predictions, which is crucial, considering that ionbot computes hundreds of millions of predictions for a typical spectrum file. 
To train and evaluate the prefix and suffix tag scoring models (Methods), the OrbitrapHCD-best and Orbitrap-HCD-TMT-10 datasets (Table 5) were split into a training and testing (5\%) set. The split was computed such that both the positive and all negative feature vectors computed from a tag are either in the training or in the testing set. GBT hyperparameters were optimized using cross-validation on the training set.

\section{The PSM scoring function}

lonbot adopts the semi-supervised learning approach pioneered by the Percolator tool 26. Each PSM in the candidate match set is encoded as a 43-dimensional feature vector in which each feature represents a different source of matching information. These features are listed in Table 4. The $36 \mathrm{X}$-ions-counts and $\mathrm{X}$-ions-explained features each consider one type of fragment ion. Combining these into one PSMs scoring function together with the other features is left to the semi-supervised the Linear Support Vector Machine (LSVM) algorithm implemented in Percolator. For the intensity-correlation feature, MS2PIP is called to predict all b- and y-ion peak intensities with the Pearson correlation between the predicted and observed intensities as the value. The RT-prederror feature is computed as the difference between the corrected observed retention time and the calibrated prediction of DeepLC. The correction is obtained by grouping the PSMs by peptidoform and then, for each group, using the retention time of the PSM with the lowest prediction error as the corrected observed retention time. As shown in the Results section, this substantially increases the relevance of the LC error feature in the PSM scoring function as it is more robust towards peptidoform with long elution windows. 
The RT-pred-error feature requires calibration to correct for different experimental conditions. This calls for a limited set of highly confident true PSMs obtained without using the feature. This is realized by first learning the PSM function without the RTpred-error feature, then selecting the 1000 highest scoring first-ranked matches to calibrate the DeepLC predictions and add the feature to the scoring function. This function is then learned again from the candidate match set.

Finally, ionbot follows the well-established concatenated target-decoy method for computing significance statistics to call true PSMs at a controlled FDR. This means that the learned PSM scoring function selects the highest scoring PSM for each spectrum, from which q-values and other statistics are computed.

Note that ionbot is not restricted to using the Percolator tool. Other Machine learning algorithms can be applied to learn the PSM scoring function as well, including unsupervised and/or non-linear models. Here, we limited ionbot to the LSVM as it is already well-established in the Proteomics community.

\section{Entrapment peptide database}

To estimate a potential matching bias, we adopted the entrapment peptides approach as described in ${ }^{27}$. Herein, shuffled target sequences are added to the target database, the entrapment peptides, that act as true target peptide. The target database is shuffled nine times to create the entrapment peptides that are added to the target database. The decoy database was constructed by shuffling the sequences in the extended target database. 


\section{MSFragger and open-pFind search settings}

We compared ionbot with MSFragger (version 3.1 in fragpipe 14.0) and open-pFind

(pFind version 3.1.5). We set the following settings equal for each search engine:

20ppm precursor mass error tolerance, peptide length in [7,30], delta-mass (mass range

allowed for unexpected modifications) in [-150Da,500Da], a maximum of two missed

cleavages, oxidation of $\mathrm{M}$ as expected and carbamidomethylation of $\mathrm{C}$ as fixed

modification. Other settings were left to the default open search settings for each

engine. 


\section{Datasets}

\section{Spectral libraries}

The Human Orbitrap-HCD-best and Orbitrap-HCD-TMT-10 spectral libraries were downloaded from the NIST Libraries of Peptide Tandem Mass Spectra ${ }^{1}$. PSMs in these libraries were filtered to contain unique peptide sequences that end with a lysine or arginine only. For peptides that are matched with different spectra, one is selected at random to avoid peptide bias. PSMs are encoded as feature vectors as described in the Methods section. Table 5 shows the number of positive and negative examples in each dataset for training the prefix and suffix tag models.

\section{Evaluation projects}

To evaluate ionbot, we selected five MS2 HCD spectrum datasets of different sizes that were obtained from different labs and with different overall experimental conditions (Table 6). One dataset (Breast) was labeled with super-SILAC and another (TMTCPTAC) with TMT10. All RAW files were downloaded from the PRIDE repository ${ }^{28}$ and converted to the Mascot MGF format using ThermoRawFileParser ${ }^{29}$ with the MS2 peak picking option enabled. Spectrum files belonging to the same sample were merged.

\footnotetext{
${ }^{1}$ https://chemdata.nist.gov
} 


\section{References}

1. Verheggen, K. et al. Anatomy and evolution of database search engines-a central component of mass spectrometry based proteomic workflows. Mass Spectrom. Rev. (2017) doi:10.1002/mas.21543.

2. Colaert, N., Degroeve, S., Helsens, K. \& Martens, L. Analysis of the resolution limitations of peptide identification algorithms. J. Proteome Res. 10, (2011).

3. Yu, F. et al. Identification of modified peptides using localization-aware open search. Nat. Commun. 11, 1-9 (2020).

4. Chi, $\mathrm{H}$. et al. Comprehensive identification of peptides in tandem mass spectra using an efficient open search engine. Nat. Biotechnol. 36, 1059-1066 (2018).

5. Bittremieux, W., Meysman, P., Noble, W. S. \& Laukens, K. Fast Open Modification Spectral Library Searching through Approximate Nearest Neighbor Indexing. J. Proteome Res. 17, 3463-3474 (2018).

6. Bouwmeester, R., Gabriels, R., Hulstaert, N., Martens, L. \& Degroeve, S. DeepLC can predict retention times for peptides that carry as-yet unseen modifications. bioRxiv 2020.03.28.013003 (2020) doi:10.1101/2020.03.28.013003.

7. Ma, C. et al. DeepRT: deep learning for peptide retention time prediction in proteomics. arXiv Prepr. arXiv1705.05368 (2017).

8. Gessulat, S. et al. Prosit: proteome-wide prediction of peptide tandem mass spectra by deep learning. Nat. Methods 16, 509-518 (2019).

9. Zeng, W.-F. F. et al. MS/MS Spectrum Prediction for Modified Peptides Using pDeep2 Trained by Transfer Learning. Anal. Chem. 91, 9724-9731 (2019).

10. Tiwary, S. et al. High-quality MS/MS spectrum prediction for data-dependent and dataindependent acquisition data analysis. Nat. Methods 16, 519-525 (2019).

11. Degroeve, S. \& Martens, L. MS2PIP: a tool for MS/MS peak intensity prediction. Bioinformatics 29, 3199-203 (2013).

12. Silva, A. S. C., Bouwmeester, R., Martens, L. \& Degroeve, S. Accurate peptide fragmentation predictions allow data driven approaches to replace and improve upon proteomics search engine scoring functions. Bioinformatics 35, (2019).

13. Käll, L., Canterbury, J. D., Weston, J., Noble, W. S. \& MacCoss, M. J. Semi-supervised learning for peptide identification from shotgun proteomics datasets. Nat. Methods 4, 923-925 (2007).

14. Keller, A., Nesvizhskii, A. I., Kolker, E. \& Aebersold, R. Empirical Statistical Model To Estimate the Accuracy of Peptide Identifications Made by MS/MS and Database Search. Anal. Chem. 74, 5383-5392 (2002).

15. Mann, M. \& Wilm, M. Error-Tolerant Identification of Peptides in Sequence Databases by Peptide Sequence Tags. Anal. Chem. 66, 4390-4399 (1994).

16. Muth, T. et al. Navigating through metaproteomics data: A logbook of database searching. Proteomics 15, 3439-3453 (2015).

17. Sticker, A., Martens, L. \& Clement, L. Mass spectrometrists should search for all peptides, but assess only the ones they care about. Nature Methods vol. 14 643-644 (2017).

18. Deutsch, E. W. et al. Universal spectrum identifier for mass spectra. bioRxiv 2020.12.07.415539 (2020) doi:10.1101/2020.12.07.415539.

19. Khoury, G. A., Baliban, R. C. \& Floudas, C. A. Proteome-wide post-translational modification statistics: Frequency analysis and curation of the swiss-prot database. Sci. Rep. 1, 1-5 (2011).

20. Gabriels, R., Martens, L. \& Degroeve, S. Updated MS²PIP web server delivers fast and 
accurate $\mathrm{MS}^{2}$ peak intensity prediction for multiple fragmentation methods, instruments and labeling techniques. Nucleic Acids Res. 47, (2019).

21. Paul Zolg, D. et al. Proteometools: Systematic characterization of 21 post-translational protein modifications by liquid chromatography tandem mass spectrometry (Ic-ms/ms) using synthetic peptides. Mol. Cell. Proteomics 17, 1850-1863 (2018).

22. Tabb, D. L., Ze-Qiang, M., Martin, D. B., Ham, A. J. L. \& Chambers, M. C. DirecTag: Accurate sequence tags from peptide MS/MS through statistical scoring. J. Proteome Res. 7, 3838-3846 (2008).

23. Devabhaktuni, A. et al. TagGraph reveals vast protein modification landscapes from large tandem mass spectrometry datasets. Nat. Biotechnol. 37, 469-479 (2019).

24. Degroeve, S., Colaert, N., Vandekerckhove, J., Gevaert, K. \& Martens, L. A reproducibility-based evaluation procedure for quantifying the differences between MS/MS peak intensity normalization methods. Proteomics 11, 1172-80 (2011).

25. Chen, T. \& Guestrin, C. Xgboost: A scalable tree boosting system. Proc. 22Nd ACM SIGKDD (2016).

26. Käll, L., Canterbury, J. D., Weston, J., Noble, W. S. \& MacCoss, M. J. Semi-supervised learning for peptide identification from shotgun proteomics datasets. Nat. Methods 4 , 923-5 (2007).

27. Granholm, V., Navarro, J. F., Noble, W. S. \& Käll, L. Determining the calibration of confidence estimation procedures for unique peptides in shotgun proteomics. $J$. Proteomics 80, 123-131 (2013).

28. Martens, L. et al. PRIDE: The proteomics identifications database. Proteomics 5, 35373545 (2005).

29. Hulstaert, N. et al. ThermoRawFileParser: Modular, Scalable, and Cross-Platform RAW File Conversion. J. Proteome Res. 19, 537-542 (2020).

30. Chick, J. M. et al. A mass-tolerant database search identifies a large proportion of unassigned spectra in shotgun proteomics as modified peptides. Nat. Biotechnol. 33, 743-749 (2015).

31. Kim, M. S. et al. A draft map of the human proteome. Nature 509, 575-581 (2014).

32. Sharma, K. et al. Cell type- and brain region-resolved mouse brain proteome. Nat. Neurosci. 18, 1819-1831 (2015).

33. Pozniak, Y. et al. System-wide Clinical Proteomics of Breast Cancer Reveals Global Remodeling of Tissue Homeostasis. Cell Syst. 2, 172-184 (2016).

34. Mertins, P. et al. Reproducible workflow for multiplexed deep-scale proteome and phosphoproteome analysis of tumor tissues by liquid chromatography-mass spectrometry. Nat. Protoc. 13, 1632-1661 (2018). 


\section{Tables}

\begin{tabular}{|l|l|l|l|l|l|}
\hline ID & Peptide & Modification & $\begin{array}{l}\text { Intensity- } \\
\text { Correlation }\end{array}$ & $\begin{array}{l}\text { RT- } \\
\text { pred- } \\
\text { error }\end{array}$ & Protein(s) \\
\hline 1 & IENNDNKPVTNSR & {$[34]$ Methyl } & 0.956 & $\begin{array}{l}8.6 \mathrm{E}-03 \\
6 \mathrm{E}-03\end{array}$ & YTHD1,YTHD3 \\
1 & IENNENKPVTNSR & - & 0.958 & $4.1 \mathrm{E}-02$ & H11 \\
\hline 2 & GGVSIAAIK & Gly-Ser[G] & 0.973 & $1.8 \mathrm{E}-03$ & H13 \\
2 & SGVSIAAIK & - & 0.98 & $1.3 \mathrm{E}-02$ & CO5 \\
\hline 3 & IEEIAAK & - & 0.904 & $2.8 \mathrm{E}-02$ & RBP2 \\
3 & IEQIAAK & - & 0.993 & $2.4 \mathrm{E}-04$ & HMGN4 \\
\hline 4 & PAPPKPEPR & - & 0.961 & $4.4 \mathrm{E}-03$ & HMGN2,HMGN3 \\
4 & PAPPKPEPK & {$[122]$ Formyl } & 0.962 & $6.6 \mathrm{E}-03$ & K1C14 \\
\hline 5 & GQVGGDVNVEMDAAPGVDISR & - & 0.82 & $1.6 \mathrm{E}-03$ & K1C16 \\
5 & GQTGGDVNVEMDAAPGVDISR & - & 0.867 & $1.5 \mathrm{E}-04$ & AHNK \\
\hline 6 & ADIDVSGPK & - & 0.92 & $2.3 \mathrm{E}-05$ & AHNK \\
6 & VPGIDATTK & - & 0.958 & $9.3 \mathrm{E}-04$ & PLEC \\
\hline 7 & IPVEVAYK & - & 0.966 & $1.1 \mathrm{E}-03$ & SRSF2,SRSF8 \\
7 & VGDVYIPR & - & 0.966 & $1.8 \mathrm{E}-02$ & DESP \\
\hline 8 & VIIQEEGTR & - & 0.991 & $3.2 \mathrm{E}-03$ & P00761 \\
8 & ISSPATINSR & {$[7]$ Deamidated } & 0.874 & $2.2 \mathrm{E}-03$ & K22O \\
\hline 9 & AQYEEIAQR & & 0.966 & $3.2 \mathrm{E}-03$ & GGCT \\
\hline 9 & AIEPNDYTGK & - & 0.956 & $5.7 \mathrm{E}-03$ & SETD2 \\
\hline 10 & NDEIIDATQK & - & 0.903 & $5.4 \mathrm{E}-02$ & BPTF \\
\hline 10 & IPGNTNVNYR & semi-tryptic & 0.966 & $3.3 \mathrm{E}-02$ & PIEZ1 \\
\hline 11 & ENIAQAVEHR & & & \\
\hline 11 & IGGYEPIFTMSAQQPSIIPFTAQAYEEISR & & & \\
\hline
\end{tabular}

Table 1 | Examples of MS2 spectra with two highly plausible co-eluting matches. Every ID in the table shows two matches for one MS2 spectrum. For each match, the table shows the peptide sequence (Peptide), its modifications to form the identified peptidoform (Modification), the intensity-correlation, the RT-pred-error (divided by the maximum retention time observed in the corresponding dataset), and the protein(s) that contain the peptide. IDs $1-5$ are examples of highly similar matches. IDs $6-11$ show matches with highly dissimilar matches. 


\begin{tabular}{|l|l|l|l|l|l|}
\hline Id & Peptide & Modification & $\begin{array}{l}\text { Intensity- } \\
\text { Correlation }\end{array}$ & $\begin{array}{l}\text { RT-pred- } \\
\text { error }\end{array}$ & Protein(s) \\
\hline 12 & FNSIPQYSPVTFDR & & 0.912 & $2.5 \mathrm{E}-02$ & BBX \\
12 & QIEAIDQIHIEYAK & & 0.755 & $8.0 \mathrm{E}-03$ & ACTN4 \\
12 & IMDPPSNEAVIISIR & [35]Oxidation & 0.947 & $1.5 \mathrm{E}-02$ & DHX36 \\
12 & IAENNIQPIFAVTSR & & 0.838 & $1.5 \mathrm{E}-03$ & ITB2 \\
\hline 13 & IISYIQR & & 0.998 & $2.2 \mathrm{E}-03$ & SAMH1 \\
13 & VAIDVGYR & & 0.976 & $1.7 \mathrm{E}-02$ & ALDR \\
13 & FYEAFSK & 0.963 & $2.9 \mathrm{E}-02$ & HS90B \\
13 & IGQGYIIK & 0.882 & $3.2 \mathrm{E}-03$ & RL3 \\
\hline 14 & ISKGANPVEIR & 0.783 & $4.9 \mathrm{E}-03$ & CH60 \\
14 & AIGDQIEGHSR & & 0.821 & $2.8 \mathrm{E}-02$ & OTUD3 \\
14 & TYYYNTETK & 0.976 & $6.6 \mathrm{E}-02$ & PR40A \\
14 & NEIPITHETK & & 0.948 & $2.0 \mathrm{E}-02$ & MALT1 \\
14 & YIEDGGIERK & & 0.926 & $2.9 \mathrm{E}-02$ & HYEP \\
\hline 15 & EYWMDPEGEMKPGR & [35]Oxidation & 0.923 & $1.2 \mathrm{E}-02$ & TCP4 \\
15 & EICNAYTEINDPMR & [4]Carbamidomethyl, & 0.752 & $4 \mathrm{E}-04$ & SYK \\
15 & TITVRDTMSDIPEVR & [35]Oxidation & 0.834 & $5.8 \mathrm{E}-02$ & DNMT1 \\
15 & FPQAITSNTPIKPSNK & & 0.923 & $2.7 \mathrm{E}-03$ & MAN1 \\
15 & MDENEFFVAVTSTNAAK & [35]Oxidation & 0.896 & $3.4 \mathrm{E}-02$ & DPYL4 \\
\hline
\end{tabular}

Table 2 | Examples of MS2 spectra (Id) with four or five highly plausible co-eluting matches. Every Id in the table shows multiple matches for one MS2 spectrum. For each match, the table shows the peptide sequence (Peptide), its modifications to form the identified peptidoform (Modification), the intensity-correlation, the RT-pred-error (divided by the maximum retention time observed in the corresponding dataset), and the protein(s) that match the peptide.

\begin{tabular}{|l|l|}
\hline Biased scoring function & Description \\
\hline b1y1-peak-count & total number of b- and y-ions matched considering only charge 1+ \\
\hline
\end{tabular}




\begin{tabular}{|l|l|}
\hline b1b2y1y2-peak-count & total number of b- and y-ions matched considering charge 1+ and 2+ \\
\hline peak -count & $\begin{array}{l}\text { total number of } \mathrm{X} \text {-ions matched with } \mathrm{X} \text { in }[\mathrm{a}, \mathrm{b}, \mathrm{b}-\mathrm{H} 2 \mathrm{O}, \mathrm{b}-\mathrm{NH} 3, \mathrm{c}, \mathrm{x}, \mathrm{y}, \mathrm{y}- \\
\mathrm{H} 2 \mathrm{O}, \mathrm{z}], \text { considering charge 1+ and 2+ }\end{array}$ \\
\hline b1y1-intensity-explained & $\begin{array}{l}\text { total explained intensity of matched b- and y-ions matched considering } \\
\text { only charge 1+ }\end{array}$ \\
\hline b1b2y1y2-intensity-explained & $\begin{array}{l}\text { total explained intensity of matched b- and y-ions matched considering } \\
\text { charge 1+ and 2+ }\end{array}$ \\
\hline intensity-explained & $\begin{array}{l}\text { total explained intensity of matched X-ions matched with X in [a,b,b- } \\
\mathrm{H} 2 \mathrm{O}, \mathrm{b}-\mathrm{NH} 3, \mathrm{c}, \mathrm{x}, \mathrm{y}, \mathrm{y}-\mathrm{H} 2 \mathrm{O}, \mathrm{z}], \text { considering charge 1+ and 2+ }\end{array}$ \\
\hline intensity-correlation & $\begin{array}{l}\text { Pearson correlation between observed and MS2PIP predicted b- and } \\
\text { y-ion intensities }\end{array}$ \\
\hline average-rank & $\begin{array}{l}\text { ranks the PSMs by the average rank for ions-count, ions-explained and } \\
\text { intensity-correlation }\end{array}$ \\
\hline
\end{tabular}

Table 3 | Biased PSM scoring functions that filter the search space. Each function considers some plausible form of matching information.

\begin{tabular}{|c|c|}
\hline Name & Description \\
\hline charge & charge state of match \\
\hline peplen & length of matched peptide \\
\hline precursor_mass & MS1 precursor mass \\
\hline num_peaks & number of peaks in the MS2 spectrum \\
\hline max_peak & intensity of highest peak in MS2 spectrum \\
\hline XY-ion-count & $\begin{array}{l}\text { number of } \mathrm{XY} \text {-ions matched with } \mathrm{X} \text { in }[\mathrm{a}, \mathrm{b}, \mathrm{b}-\mathrm{H} 2 \mathrm{O}, \mathrm{b}-\mathrm{NH} 3, \mathrm{c}, \mathrm{x}, \mathrm{y}, \mathrm{y}-\mathrm{H} 2 \mathrm{O}, \mathrm{z}] \text { and } \mathrm{Y} \\
\text { charge state }+1 \text { or }+2\end{array}$ \\
\hline $\begin{array}{l}\mathrm{XY} \text {-ion- } \\
\text { explained }\end{array}$ & $\begin{array}{l}\text { total explained intensity for the } X Y \text {-ions matched with } X \text { in }[a, b, b-H 2 O, b- \\
N H 3, c, x, y, y-H 20, z] \text { and } Y \text { charge state }+1 \text { or }+2\end{array}$ \\
\hline $\begin{array}{l}\text { intensity- } \\
\text { correlation }\end{array}$ & $\begin{array}{l}\text { Pearson correlation between observed and MS2PIP predicted b- and y-ion } \\
\text { intensities }\end{array}$ \\
\hline RT-pred-error & difference between corrected observed and DeepLC predicted retention time \\
\hline
\end{tabular}

Table 4| The $\mathbf{4 3}$ features used in the ionbot PSM scoring function feature vector. Note that XY-ioncount and $\mathrm{XY}$-ion-explained each constitute 18 different features.

\begin{tabular}{|l|l|l|l|}
\hline Dataset & Version & \#Positives & \#Negatives \\
\hline Orbitrap-HCD-best & $05-19-2020$ & 212.761 & 42.531 .154 \\
\hline Orbitrap-HCD TMT-10 & $10-30-2019$ & 318.972 & 63.794 .400 \\
\hline
\end{tabular}


Table 5: Spectral libraries used to train the prefix and suffix models. For each library the number of computed feature vectors labeled as positive (\#Positives) and computed feature vectors labeled as negative (\#Negative) is show. Libraries were downloaded from chemdata.nist.gov.

\begin{tabular}{|l|l|l|l|l|}
\hline Name & Accession & $\begin{array}{l}\text { \#MS/MS } \\
\text { spectra }\end{array}$ & Sample & Machine \\
\hline HEK293 & PXD001468 & 1.121 .149 & HEK293 cell & Orbitrap Q-Exactive \\
\hline CD8T & PXD000561 & 406.913 & CD8T cell & Orbitrap Elite \\
\hline Brain & PXD001250 & 669.350 & Mouse brain & Orbitrap Q-Exactive \\
\hline Breast & PXD000815 & 382.022 & $\begin{array}{l}\text { Breast cancer } \\
\text { tumor }\end{array}$ & Orbitrap Q-Exactive \\
\hline TMTCPTAC & PXD012703 & 1.064 .359 & Tumor tissue & $\begin{array}{l}\text { Orbitrap Q-Exactive } \\
\text { Plus }\end{array}$ \\
\hline
\end{tabular}

Table 6: Evaluation datasets used in the Results section. The first four datasets were downloaded from the PRIDE repository (Accession). The TMT datasets was downloaded from the CPTAC portal. 


\section{Figures}

(a)

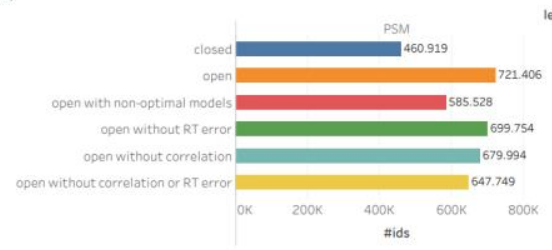

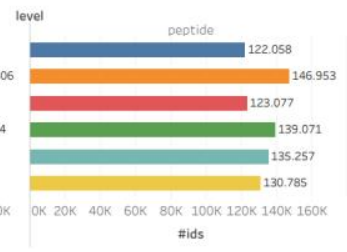
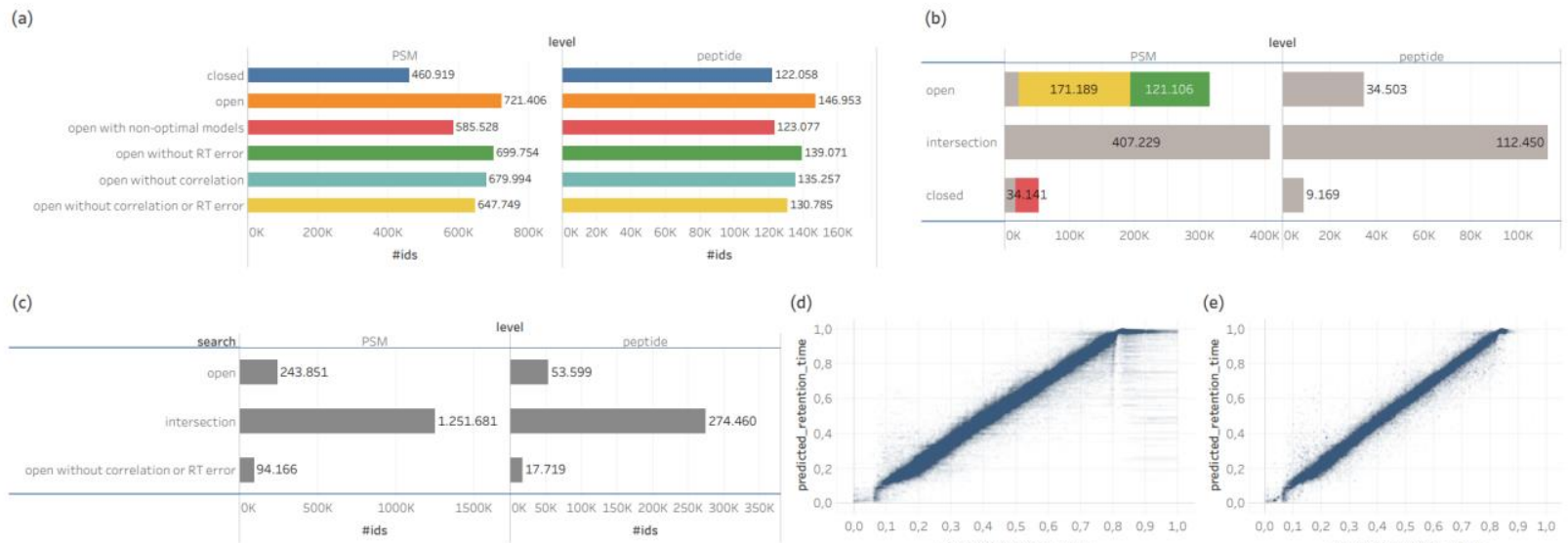

(d)
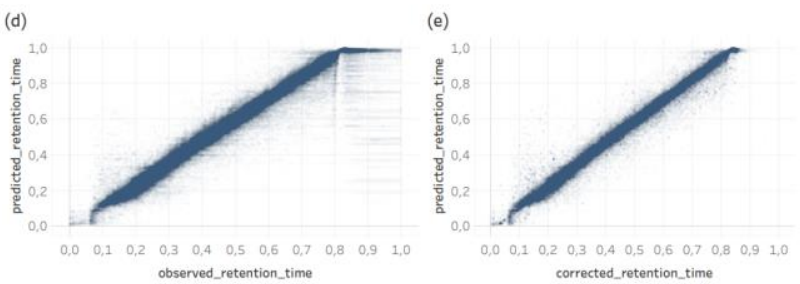

(f)

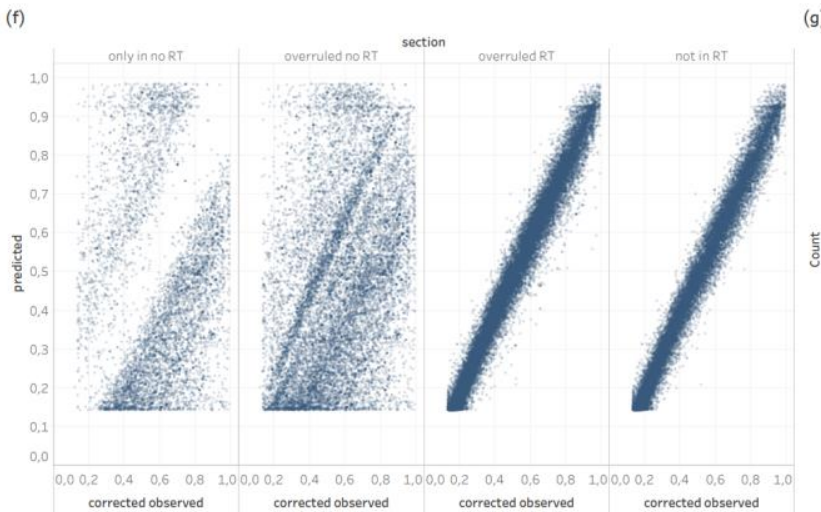

(g)

(h)

(i)
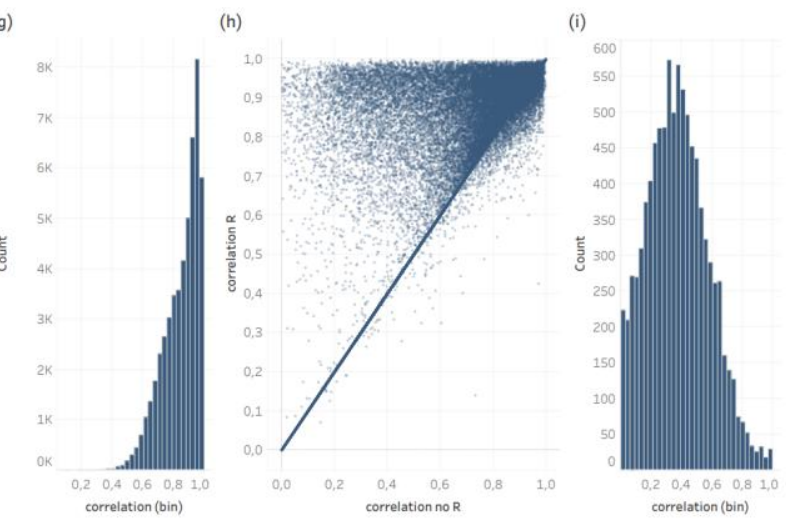

Fig. 1 | Comparing different implementations of the ionbot search engine. (a) shows the number of identifications in HEK239 for the different implementations. These are a closed search (dark blue), a standard open search (orange), an open search using non-optimal models (red) (for the TMTCPTAC dataset, models (tag-models, MS2PIP and DeepLC) trained on unlabeled HCD were used, while for all other datasets models trained on TMT labeled data were used), an open search without using the RTpred-error feature (green), an open search without using the intensity-correlation feature and biased scoring function (light blue), and an open search without intensity-correlation and RT-pred-error (yellow). (b) shows the identification overlap between a closed and open HEK239 search. For the closed searches, spectra with overruled identifications are shown in red. For open searches, matches with an unexpected modification are shown in green, while wide error matches are shown in yellow. (c) shows the identification overlap between an open HEK239 search and the same open search without using the intensity-correlation and RT-pred-error information. (d) true observed versus DeepLC predicted retention time for CD8T. (e) corrected observed versus DeepLC predicted retention time for CD8T. (f) compares corrected observed versus predicted retention times for HEK239 searches using (with-RT) and not using (no-RT) the RT-pred-error feature. (g) shows intensity-correlation (R) for PSMs identified in HEK239 identified with using $R$ but not identified not using $R$ (no $R$ ). (h) plots the intensity-correlations for spectra identified in $R$ and no $R$, but with a different match. (i) shows $R$ for PSMs identified in no $R$ but not identified in $\mathrm{R}$. 
(a)

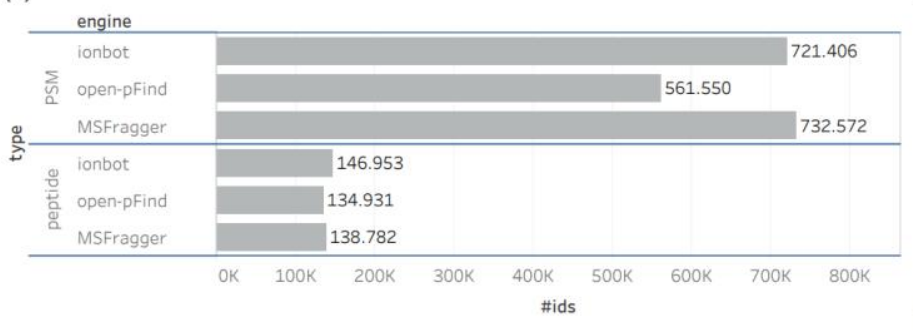

(b)

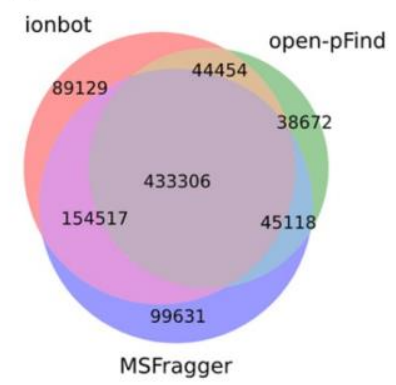

(c)

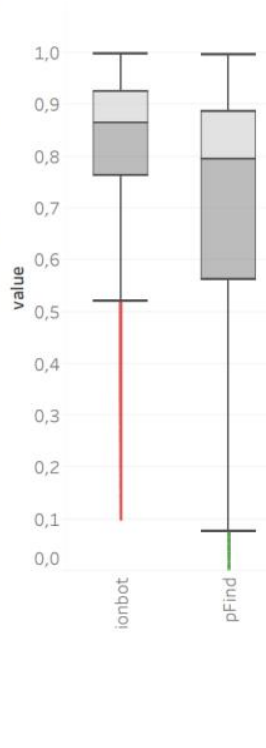

engine

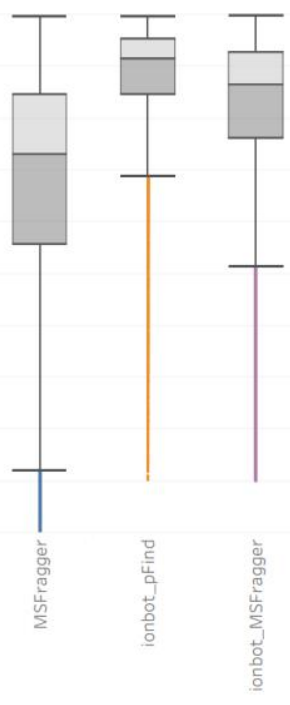

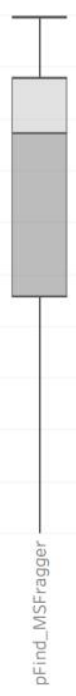

Fig. 2 | Identification comparison between ionbot, open-pFind and MSFragger for HEK239. (a) shows the number of PSM and unique peptide identifications for each engine. (b) shows the identification overlap between the three open search engines at PSM level (left), and at unique peptide sequences level (right). (c) shows the value of the intensity-correlation feature for matches in the different sections of the venn diagrams shown (b). The color of the outliers match the colors of the different sections. Outliers with value smaller than zero are not shown. 
(a)

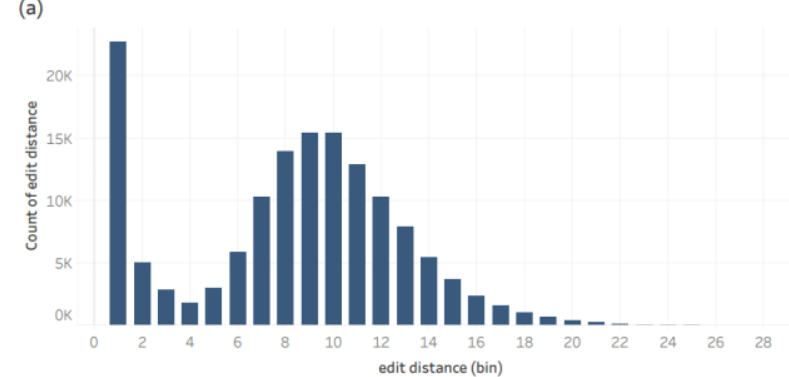

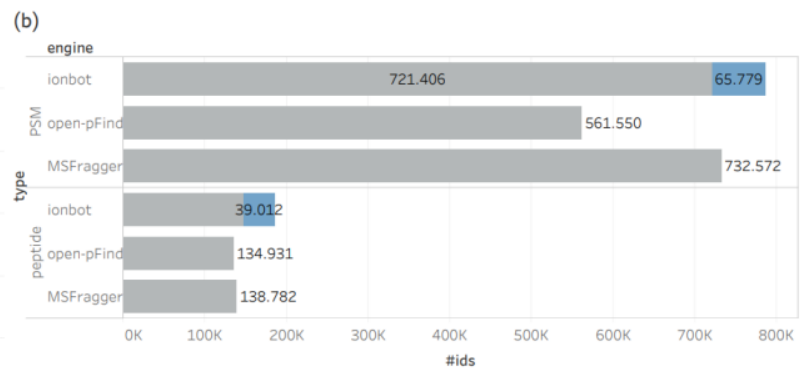

(c)

(c) (d)

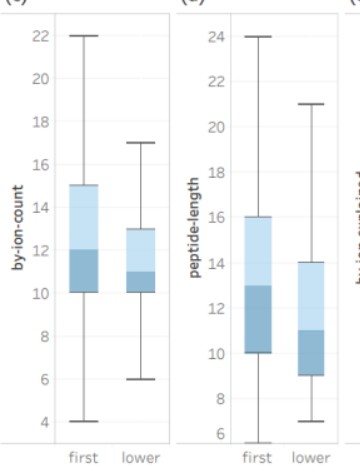

(e)

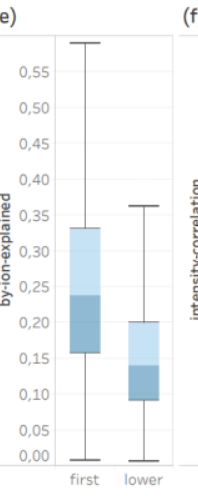

(f)

(g)

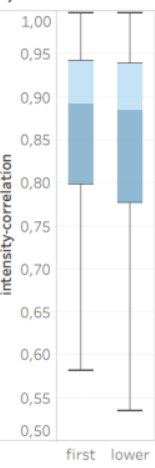

(g)

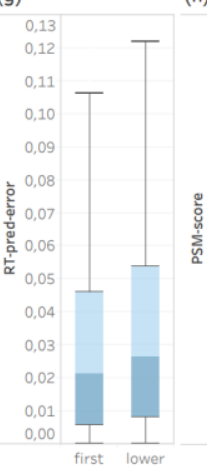

(h)

(i)

Fig. 3 | Evaluating co-eluting matches in HEK239. (a) shows the edit distance between two identified matches in one MS2 spectrum. Only MS2 spectra with two identified matches are shown. (b) shows the same identification counts as Fig. 2a with the lower-ranked co-eluting matches (edit distance $>2$ ) added in blue. The next six boxplots compare different sources of matching information between the first-ranked and lower-ranked co-eluting matches. This information is (c) the number of b-and $y$-ion peaks matched, (d) the length of the matched peptide, (e) the total explained intensity of the b- and y-ion peaks matched, (f) the intensity-correlation, (g) the RT-pred-error and (h) the PSM score. (i) shows the percentage of the lower-ranked matches identified as first-ranked PSM in both MSFragger and open-pFind (green), only open-pFind (orange), and only MSFragger (blue). In (j), for the lower-ranked PSMs unique to ionbot (yellow section in (i)), the percentage of unique peptide matches identified as first-ranked PSM (from another MS2 spectrum) in both MSFragger and open-pFind (green), only open-pFind (orange), and only MSFragger (blue) is shown. 